\title{
THE SUBSTANCE VIEW: A CRITIQUE (PART 2)
}

\section{ROB LOVERING}

\author{
Keywords \\ substance view, \\ intrinsic value, \\ moral standing, \\ human fetus, \\ essential property, \\ rational moral agency, \\ potential
}

\begin{abstract}
In my initial critique of the substance view, I raised reductio-style objections to the substance view's conclusion that the standard human fetus has the same intrinsic value and moral standing as the standard adult human being, among others. In this follow-up critique, I raise objections to some of the premises invoked in support of this conclusion. I begin by briefly presenting the substance view as well as its defense. (For a more thorough presentation, see the first part of my critique.) I then raise objections to three claims involved in the substance view's defense: the claim that the standard human fetus's intrinsic value and moral standing is a function of its potentiality; the claim that the standard human fetus's intrinsic value and moral standing is a function of its essential properties; and the claim that it is the possession of the basic potential for rational moral agency that best accounts for the wrongness of killing the standard human fetus, among others.
\end{abstract}

\section{INTRODUCTION}

In my initial critique of the substance view, I raised reductio-style objections to the substance view's conclusion that the standard human fetus has the same intrinsic value and moral standing as the standard adult human being, among others. ${ }^{1}$ In this follow-up critique, I raise objections to some of the premises invoked in support of this conclusion. I begin by briefly presenting the substance view as well as its defense. (For a more thorough presentation, see the first part of my critique.) I then raise objections to three claims involved in the substance view's defense: the claim that the standard human fetus's intrinsic value and moral standing is a function of its potentiality; the claim that the standard human fetus's intrinsic value and moral standing is a function of its essential properties; and the claim that it is the possession of the basic potential for rational moral agency that best accounts for the wrongness of killing the standard human fetus, among others.

${ }^{1}$ R. Lovering. The Substance View: A Critique. Bioethics 2012. DOI: 10.1111/j.1467-8519.2011.01954.x.

\section{ON THE SUBSTANCE VIEW}

When it comes to determining the intrinsic value and moral standing of the standard human fetus and, specifically, whether it is prima facie seriously wrong to kill it (hereafter, simply 'wrong'), contemporary philosophers often rely upon arguments from inference to the best explanation. ${ }^{2}$ For example, some philosophers first judge that it is wrong to kill the following individuals: (1) the standard adult human being (including those who are sleeping), (2) the reversibly comatose adult human being, (3) the suicidal adult human being, and (4) the standard human infant, among others. Next, they attempt to determine what property or set of properties - accidental or essential - the possession of which is sufficient for the wrongness of killing them. Once that's been done, they attempt to determine whether another individual, (5) the

\footnotetext{
2 By 'human fetus', I mean the developing human organism from conception until birth. By 'intrinsic' value I mean value it's logically possible for something to have even if it were the only thing that existed. By 'moral standing' I mean the property of being morally considerable, a property in virtue of which moral agents have moral obligations toward those things that possess it.
} 
standard human fetus, possesses that property or set of properties. If it does, they reason, then just as it is wrong to kill individuals (1) - (4), so it is wrong to kill individual (5).

Adopting such an approach, defenders of the substance view (SV) hold that the property the possession of which is sufficient for the wrongness of killing individuals (1) - (4) is the essential property of being a person or, more specifically, a rational moral agent. ${ }^{3}$ Moreover, they contend that, like individuals (1) - (4), individual (5), the standard human fetus, possesses the essential property of rational moral agency as well. Accordingly, they reason, it is just as wrong to kill individual (5) as it is to kill individuals (1) - (4). ${ }^{4}$

\section{The substance view on rational moral agency}

According to defenders of SV, being a rational moral agent involves possessing certain potentialities, such as the potential to reason, the potential to think in terms of and regulate one's behavior in accordance with moral principles, and so on. ${ }^{5}$ However, they contend that these potentialities need not be immediately actualizable for something to possess the essential property of rational moral agency. You see, defenders of SV distinguish among three types of potential, to be referred to here as basic, proximate, and ultimate. A basic potential for $\mathrm{X}$ is an active but not remotely actualizable potential for X. A proximate potential for $\mathrm{X}$ is an active and actualizable potential for $\mathrm{X}$ but, for whatever reason, is not immediately actualizable at a given time. And an ultimate potential for $\mathrm{X}$ is an active and immediately actualizable potential for X. ${ }^{6}$ Given these distinctions, we may now see on what grounds defenders of SV believe that the

\footnotetext{
${ }^{3}$ P. Lee \& R.P. George. 2007. Body-Self Dualism in Contemporary Ethics and Politics. Cambridge, UK: Cambridge University Press; P. Lee \& R.P. George. The Nature and Basis of Human Dignity. Ratio Juris 2008; 21(2): 179-193; Patrick Lee. The Pro-Life Argument from Substantial Identity: A Defense. Bioethics 2004; 18(3): 249-263; P. Lee \& R.P. George. The Wrong of Abortion. 2005. In Contemporary Debates in Applied Ethics. A.I. Cohen \& C.H. Wellman, eds. Oxford, UK: Blackwell Publishing: 13-26; R.P. George \& C. Tollefsen. 2008. Embryo: A Defense of Human Life. New York, NY: Doubleday; F. Beckwith. 2007. Defending Life: A Moral and Legal Case Against Abortion Choice. New York, NY: Cambridge University Press.

${ }^{4}$ Given the preceding, one might wonder why this view is referred to as the 'substance view.' Simply put, defenders of the substance view hold that rational moral agency is an essential property of a particular substance sort - the human organism - and that individuals (1) - (5) are essentially human organisms.

${ }^{5}$ As discussed in my first critique, defenders of SV use 'potential' and 'capacity' interchangeably.

${ }^{6}$ For the sake of space, I must forego discussing the difference between active and passive potentialities. For such a discussion, see M. Tooley, et al. 2009. Abortion: Three Perspectives. New York, NY: Oxford University Press: 38.
}

standard human fetus possesses the essential property of rational moral agency. According to defenders of $\mathrm{SV}$, in order for something to possess the essential property of rational moral agency, one need not possess the ultimate or even proximate potential for rational moral agency one need only possess the basic potential for rational moral agency. And it is just this basic potential that the standard human fetus possesses.

\section{SV's defense}

With the preceding in mind, we may now state SV's defense precisely.

To begin with, defenders of SV believe that intrinsic value is not a degreed property - 'you either have it or you don't. ${ }^{7}$ In turn, they hold that the intrinsic value and moral standing of individuals (1) - (4) must be a function of essential rather than accidental properties, as the latter admit of degrees. ${ }^{8}$ For, if the intrinsic value and moral standing of individuals (1) - (4) were a function of accidental and thereby degreed properties, then they would not possess the same intrinsic value and moral standing since they do not share all the same accidental properties, let alone share them to the same degree. But individuals (1) - (4) do possess the same intrinsic value and moral standing, or so defenders of SV believe. Thus, intrinsic value is not an accidental, degreed property; in turn, the intrinsic value and moral standing of individuals (1) - (4) must be a function of essential properties. ${ }^{9}$

With this said, defenders of SV argue that possessing the essential property of the basic potential for rational moral agency is sufficient for the intrinsic value of individuals (1) - (4) and, in turn, the wrongness of killing them. To see this, consider a theory of intrinsic value and moral standing that emphasizes possessing, say, the accidental property of the ultimate potential for rational moral agency. Individuals (2) and (4) lack the accidental property of the ultimate potential for rational moral agency, as do individuals (1) and (3) on occasion (such as when they are asleep). But, it's counterintuitive if not absurd to think that - as a result their lacking this accidental property - it is not wrong to kill them. SV, on the other hand, accounts for the judgment that it is wrong to kill them, since each possesses the essential property of the basic potential for rational moral agency. Moreover, like individuals (1) - (4), individual (5), the standard human fetus, has the essential property of the basic

\footnotetext{
${ }^{7}$ Beckwith, op. cit. note 3, p. 139.

${ }^{8}$ Briefly, $\mathrm{P}$ is an essential property of $\mathrm{X}$ if $\mathrm{X}$ cannot be what it is without $\mathrm{P}$; whereas $\mathrm{P}$ is an accidental property of $\mathrm{X}$ if $\mathrm{X}$ can be what it is without P.

${ }^{9}$ George and Tollefsen, op. cit. note 3, pp. 117ff; Lee, op. cit. note 3, pp. $253 \mathrm{ff}$.
} 
potential for rational moral agency. Accordingly, it is just as wrong to kill individual (5) as it is to kill individuals (1) $-(4)$.

\section{OBJECTIONS}

Before moving on to objections, a couple of things should be noted. First, as mentioned above, the objections to be raised here pertain only to premises invoked in support of SV's conclusion that the standard human fetus has the same intrinsic value and moral standing as the standard adult human being, among others. (For objections to SV's conclusion itself, see my first critique.) Second, throughout the following, unless otherwise noted, statements such as 'one ought to save the ten frozen human embryos rather than the five-year-old girl' or 'it is just as wrong to kill the standard human fetus as it is to kill individuals (1) - (4)' are to be understood as all-elsebeing-equal claims.

\section{On intrinsic value and moral standing being a function of potentiality}

The first objection pertains to SV's claim that the intrinsic value and moral standing of the human fetus is a function of its potentiality. Now, a lot of ink has been spilled debating the moral importance of the human fetus's potentiality, whether for rational moral agency, personhood, or other. ${ }^{10}$ Even so, I would be remiss not to include at least a couple of criticisms of the view that the human fetus's basic potential for rational moral agency carries moral weight, even if only briefly. The first criticism is raised here for the first time; accordingly, defenders of SV have not had the opportunity to respond to it. The second criticism, however, has been raised before, and defenders of SV have responded to it. This, in turn, allows me to criticize their response.

It would be prudent to begin this discussion with the following: from the mere fact that a given property, $p$, is shared by individuals (1) - (4), it does not immediately follow that $p$ confers moral standing; nor does it follow that an explanation of the wrongness of killing individuals (1) - (4) that turns on $p$ is a plausible theory of moral standing. To see this, consider the following explanation of the wrongness of killing individuals (1) - (4): individuals (1) - (4) share the property of being bipedal. Though it is true that individuals (1) - (4) share this property, most would agree that this property does not confer moral standing and, in turn, that an explanation of

\footnotetext{
${ }^{10}$ See, for example, M. Tooley. 1983. Abortion and Infanticide. New York, NY: Oxford University Press; and J. McMahan. 2002. The Ethics of Killing. New York, NY: Oxford University Press.
}

the wrongness of killing of individuals (1) - (4) that turns on possession of this property is not a plausible theory of moral standing.

That said, even if individuals (1) - (4) all possess the basic potential for rational moral agency, it does not immediately follow that the basic potential for rational moral agency confers moral standing or that an explanation of the wrongness of killing of individuals (1) - (4) that turns on possession of this property is a plausible theory of moral standing. Two questions, then, arise. First, does the basic potential for rational moral agency confer moral standing? And, second, even if it does, is an explanation of the wrongness of killing individuals (1) - (4) that turns on this property a plausible theory of moral standing?

\section{Does the basic potential for rational moral agency confer moral standing?}

Regarding the first question, there are a number of reasons for thinking that the basic potential for rational moral agency does not confer moral standing. The first reason may be elicited through a discussion of human infants that have died within a year of their birth due to congenital heart disease. Given that their deaths were the result of heart disease and not a neurological disorder, it seems safe to say that intrinsic to these infants were the plans for the proximate and ultimate potential for rational moral agency, albeit plans that were never realized due to congenital heart disease. In other words, it seems safe to say that these infants possessed the basic potential for rational moral agency. (The word 'seems' here is operative and, thus, this claim will be scrutinized shortly.) Since possession of the basic potential for rational moral agency occurs at conception (or very soon thereafter), the human fetuses that developed into these infants possessed the basic potential for rational moral agency as well.

With the preceding in mind, suppose that, sometime in the future, all human infants are born with congenital heart disease and die within one year of their birth. Just as with the infants that have already died as a result of congenital heart disease, it seems safe to say that intrinsic to these future human infants is the plans for the proximate and ultimate potential for rational moral agency, albeit plans that are never realized due to congenital heart disease. In other words, it seems safe to say that these future human infants possess the basic potential for rational moral agency. (Again, the word 'seems' here is operative and this claim will be scrutinized shortly.) And, since possession of the basic potential for rational moral agency occurs at conception (or very soon thereafter), the future human fetuses which develop into these future human infants possess the basic potential for rational moral agency as well. 
Or do they? After all, in not a single case do any of these future human fetuses come to possess the proximate or ultimate potential for rational moral agency. Given this, some might argue that none has the basic potential for rational moral agency. Of course, others might disagree, arguing that, despite the fact that none of these future human fetuses come to possess the proximate or ultimate potential for rational moral agency, each nevertheless possesses the basic potential for rational moral agency. Rather than taking sides in this debate, I will argue that, either way, there are serious problems for SV.

Regarding the view that these future human fetuses $d o$ possess the basic potential for rational moral agency, there are at least two problems. As indicated above, it is one thing for an entity to possess a given property; it's another thing for the possession of that property to confer moral standing. On the view under consideration, these future human fetuses possess the basic potential for rational moral agency. However, it is a potential that in no case actualizes; it is a potential that never progresses to the point of the proximate or ultimate potential for rational moral agency. And it's very difficult to see how this unactualizable potential could confer moral standing. For all practical purposes, there is no difference between possessing this unactualizable potential and not possessing it at all. Given this, it's very difficult to see how there could be a moral difference between possessing this unactualizable potential and not possessing it at all.

Second, when combined with SV, the view that these future human fetuses possess the basic potential for rational moral agency entails that it would be just as wrong to kill one of these future human fetuses - fetuses whose basic potential for rational moral agency never actualizes - as it would be to kill a standard adult human being. And this is strongly counterintuitive if not absurd.

As for the view that these future human fetuses do not possess the basic potential for rational moral agency, a question arises: Why not? The most obvious answer is that not one of them ever develops to the point wherein they possess the proximate or ultimate potential for rational moral agency.

But, first, not developing to the point wherein one possesses the proximate or ultimate potential for rational moral agency does not immediately entail that one lacks the basic potential for rational moral agency. Potentialities can be possessed, of course, even when they are not fully actualized.

Second, if the view that these future human fetuses do not possess the basic potential for rational moral agency is supported on these grounds - that not one of them ever develops to the point wherein they possess the proximate or ultimate potential for rational moral agency - another question arises: What percentage of human fetuses must develop to the point wherein they possess the proximate or ultimate potential for rational moral agency in order for it to be the case that human fetuses (present or future) possess the basic potential for rational moral agency? Arguably not $100 \%$, as that would entail (implausibly) that human fetuses do not possess the basic potential for rational moral agency; a conclusion, of course, defenders of SV would be loath to accept. Then again, arguably not, say, $10 \%-20 \%$, since to hold that human fetuses possess the basic potential for rational moral agency on the grounds that a small percentage of them develop to the point wherein they possess the proximate or ultimate potential for rational moral agency is strikingly arbitrary.

It seems, then, that a large percentage (say, somewhere between $75 \%$ and $95 \%$ ) is required if one is to hold that human fetuses possess the basic potential for rational moral agency. If this is indeed the case, then a problem arises for SV. For, conservatively, it's estimated that $60 \%$ of pregnancies spontaneously abort. ${ }^{11}$ What this tells us is that, at best, only $40 \%$ of human fetuses develop to the point wherein they possess the proximate or ultimate potential for rational moral agency. So, if $75 \%$ to $95 \%$ of human fetuses must develop to the point wherein they possess the proximate or ultimate potential for rational moral agency in order for it to be the case that human fetuses possess the basic potential for rational moral agency, then human fetuses lack the basic potential for rational moral agency. In turn, given SV, they lack full moral standing - once again, a conclusion defenders of SV would be loath to accept.

Of course, one could respond to the preceding by dropping the percentage of human fetuses that must develop to the point wherein they possess the proximate or ultimate potential for rational moral agency to 40 (or lower). But that one could do so in a principled way is doubtful. Moreover, and similar to the point made above, to hold that human fetuses possess the basic potential for rational moral agency on the grounds that a minority of them develop to the point wherein they possess the proximate or ultimate potential for rational moral agency would be strikingly arbitrary. And, finally, the lower one drops the percentage - that is, the closer one gets to $0 \%$ - the closer one moves to a rejection of the very grounds currently invoked in order to defend the view these future human fetuses do not possess the basic potential for rational moral agency, namely, that not one of them ever develops to the point wherein they possess the proximate or ultimate potential for rational moral agency.

A second reason to think that the basic potential for rational moral agency does not confer moral standing one to which defenders of SV have replied - may be elicited by considering temporarily comatose individuals.

\footnotetext{
${ }^{11}$ C.E. Boklage. Survival Probability of Human Conceptions from Fertilization to Term. International Journal of Fertility 1990; 35(2): 75-94; Henri Leirdon. 1977. Human Fertility: The Basic Components. Chicago, IL: University of Chicago Press.
} 
Most people - including defenders of SV, of course believe that it is wrong to kill individuals who are reversibly comatose. Yet, as defenders of SV see it, this presents a problem for some defenders of abortion, namely, those who deny that the fetus's potential for higher mental functions confers moral standing. For 'they will have to concede that reversibly comatose human beings will be persons because they have the potentiality or capacity for higher mental functions. ${ }^{12}$

Yet, that it is simply the potential for higher mental functions that renders it immoral to kill reversibly comatose individuals is subject to reductio. As Michael Tooley writes, in such cases:

it does not matter whether the person will come out of the coma without assistance, or whether some medical intervention is needed for the person to recover. The fact, for example, that the person could emerge from the coma only if an operation to relieve pressure on the person's brain would not make it permissible to kill the person. ${ }^{13}$

Given this, Tooley maintains, it is clear that, to the extent that potentialities are relevant to an entity's moral standing, passive potentialities are just as relevant as active potentialities. ${ }^{14}$ But this, when combined with SV, leads to an absurdity. Specifically, if potentialities are relevant to an entity's moral standing and, in turn, passive potentialities are just as relevant as active potentialities, then, given SV, every one of the trillion-plus somatic cells that make up a human organism would have the same intrinsic value and moral standing as individuals (1) - (4). For, given human cloning by somatic cell nuclear transfer (SCNT), every one of them has the passive potential for rational moral agency. In turn, when one scratches one's nose and thereby kills any number of one's somatic cells, one commits murder. But this is absurd.

George and Tollefsen have challenged the preceding type of argument by maintaining that somatic cells that undergo SCNT do not survive SCNT - that the identity relation between pre-SCNT cells and the post-SCNT cells does not obtain. ${ }^{15}$ If this is the case, then whatever potential the original somatic cell may have had before it underwent SCNT, it is destroyed by SCNT and, in turn, somatic cells involved in SCNT do not have a passive potential for rational moral agency.

Yet, it's not clear that the identity relation between pre-SCNT cells and the post-SCNT cells fails to obtain. ${ }^{16}$

\footnotetext{
12 Lee and George 2007, op. cit. note 3, p. 135.

13 Tooley et al., op. cit. note 6, p. 38.

14 Ibid: $37-40$.

${ }^{15}$ George and Tollefsen, op. cit. note 3, pp. 163ff.

${ }^{16}$ I have made this point elsewhere. See Rob Lovering. Futures of Value and the Destruction of Human Embryos. Canadian Journal of Philosophy 2009; 39(3): 463-488, endnote \#35.
}

To motivate this point, consider the issue of personal identity. Most philosophers - including defenders of SV think that much of the human organism can be destroyed (arms, legs, ears, eyes, etc.) without thereby losing personal identity. And some philosophers think that all that's needed for the preservation of personal identity is preservation of the upper brain. With this in mind, preservation of just the nucleus of a given somatic cell may be sufficient for the identity relation to obtain between preand post-SCNT cells. Until someone establishes necessary and sufficient conditions for somatic-cell identity, we cannot say with confidence whether the identity relation between pre-SCNT cells and the post-SCNT cells fails to obtain. Accordingly, it's not at all clear that somatic cells do not survive SCNT, and the objection remains in play.

\section{Is an explanation that turns on the basic potential for rational moral agency a plausible theory of moral standing?}

Let us now turn our attention to the second question, namely, even if the basic potential for rational moral agency is moral-standing-conferring, is an explanation of the wrongness of killing individuals (1) - (4) that turns on this property a plausible theory of moral standing? Defenders of SV think so, of course, on the basis of its explanatory power: no other view, they contend, can explain the wrongness of killing individuals (1) - (4) as well as SV. But even if one grants (charitably) that no other view can explain the wrongness of killing individuals (1) - (4) as well as SV, it does not immediately follow that SV is a plausible theory of moral standing, all things considered. For it may nevertheless be undermined by counterintuitive implications, among other things. And, if what I have argued in my first critique of SV and what I am arguing here is correct, then SV is in fact undermined by counterintuitive implications.

To motivate this point, consider that even defenders of SV concede that a theory of moral standing may be razed by burdensome, counterintuitive implications. Take, for example, the theory of moral standing according to which it is wrong to kill individuals that possess the proximate or ultimate potential for rationality. This theory entails that it is wrong to kill individuals (1) - (3) but not (4), the standard human infant. Now, defenders of SV deem this theory implausible on the grounds that it cannot account for the intuition that the killing of infants is just as wrong as the killing of individuals (1) - (3). So in one fell intuitive swoop, as it were, defenders of SV reject theories of moral standing that cannot account for the intuition that infanticide is just as wrong as killing individuals (1) - (3).

But, retaining the intuition that infanticide is wrong via $\mathrm{SV}$ requires that one adopt yet other positions on moral 
matters that are strongly counterintuitive. Given this, determining the plausibility of SV involves weighing the intuition of the wrongness of infanticide against these other counter-intuitions, among other things. And if the counter-intuitions are strong enough and numerous enough to outweigh the infanticide intuition, then SV is all-things-considered implausible. To raise just one example from my first critique, an implication of SV's conclusion that the standard human fetus has the same moral standing as individuals (1) - (4) is that, if one has to choose between saving the life of a five-year-old girl and ten frozen human embryos, one ought to save the ten frozen human embryos. After all, saving ten intrinsically valuable beings is clearly better than saving only one when all parties are equally intrinsically valuable (as all these parties are, according to SV). But that one ought to save the ten frozen human embryos rather than the fiveyear-old girl is strongly counterintuitive if not absurd.

All this to say, even if one grants that no other explanation can explain the wrongness of killing individuals (1) - (4) as well as SV, it does not immediately follow that SV is a plausible theory of intrinsic value and moral standing, all things considered. For it may be undermined by other counterintuitive implications, such as those addressed here as well as in my first critique of SV.

\section{On intrinsic value and moral standing being a function of essential properties}

The next objection pertains to SV's claim that the human fetus's intrinsic value and moral standing must be a function of essential rather than accidental properties and, thus, that intrinsic value does not admit of degrees. This claim is objectionable on numerous grounds.

Regarding moral standing being a function of essential rather than accidental properties, suppose that a rationalmoral-agency serum has been developed and injected into a number of chimpanzees. ${ }^{17}$ As a result, a year later the chimpanzees come to possess the ultimate potential for rational moral agency. Accordingly, they behave as other beings with the ultimate potential for rational moral agency (such as ourselves) do: they reason, praise, console, reprimand, etc. They are, in short, real-life versions of the apes from Planet of the Apes. Now, clearly, the ultimate potential for rational moral agency in their case would be an accidental property; but just as clearly, these chimpanzees would have the same moral standing as any other being with the ultimate potential for rational moral agency. That their ultimate potential for rational moral agency is an accidental property seems utterly

\footnotetext{
17 The idea of a rational moral agency serum is borrowed from Tooley, who has been using it for decades. For a more recent example, see Tooley, et al. op. cit. note 6, p. 47.
}

inconsequential. (Imagine informing these chimpanzees that they do not have a right to life, for example, since, unfortunately, their ultimate potential for rational moral agency is accidental rather than essential in nature. 'What difference does that make?' would no doubt be their response, and rightfully so.) Of course, such chimpanzees would not be members of the species Homo sapiens, but even defenders of SV recognize that belonging to the species Homo sapiens is not necessary for the ultimate potential for rational moral agency and, with it, full moral standing. ${ }^{18}$ All this is to say that, despite the fact that their ultimate potential for rational moral agency would be an accidental property, such chimpanzees would nevertheless possess the same moral standing as any other being with the ultimate potential for rational moral agency. It's not the case, then, that an entity's moral standing must be a function of its essential properties.

Regarding intrinsic value not admitting of degrees, one wonders what defenders of SV think about the value of non-human animals, particularly those (dolphins, whales, chimpanzees) who - though lacking the ultimate potential for rational moral agency - are nevertheless relevantly similar to those who possess it. ${ }^{19}$ Though they have explicitly addressed the issue of the moral standing of non-human animals to some extent - arguing, specifically, that non-human animals do not have full moral standing ${ }^{20}$ - they have not (to my knowledge) explicitly addressed whether non-human animals have value and, if so, whether their value is intrinsic or extrinsic in nature.

Now, it's very hard to believe that animals such as dolphins, whales, and chimpanzees are not valuable at all. Assuming they are, then, the question is whether their value is intrinsic or extrinsic in nature. If defenders of SV are correct and intrinsic value is not a degreed property ('you either have it or you don't'), then if such animals have intrinsic value, they have it to the same extent that standard adult human beings do. But this is counterintuitive, and certainly no defender of SV would accept this. However, if they don't have intrinsic value, that leaves them with having mere extrinsic value, and it's equally counterintuitive that the value of dolphins, whales, and chimpanzees is strictly extrinsic, particularly

\footnotetext{
${ }^{18}$ See Lee and George 2008, op. cit. note 3, 176. Beckwith writes, ‘. . . if another species exists, whether in this world or in another ... which possesses a personal nature from the moment any of its individual members come into being, then pro-lifers would seek to have these creatures protected from unjustified homicide as well' (Beckwith: 161-62).

${ }^{19}$ Indeed, so much so that even scientists are now calling for recognizing dolphins and whales as 'non-human persons.' See http://www. telegraph.co.uk/science/science-news/9093407/Dolphins-should-berecognised-as-non-human-persons.html.

${ }^{20}$ See, for example, Lee and George 2007, op. cit. note 3, ch 2, section 3 .
} 
given their similarities to beings with the ultimate potential for rational moral agency. This is precisely why many philosophers reject the view that intrinsic value is not a degreed property and, instead, hold that intrinsic value comes in degrees as determined by accidental-property possession. ${ }^{21}$

Defenders of SV offer two related but distinct reasons for thinking that intrinsic value does not admit of degrees. First, they argue that, if intrinsic value admitted of degrees, then there would be no non-arbitrary way of designating which entities have full moral standing and which do not, as there would be no non-arbitrary reason for selecting one degree of accidental-property possession as full-moral-standing-conferring over another.

The problem with this reply is that it trades on an ambiguity, for there are at least two different senses of 'non-arbitrary'. ${ }^{22}$ One sense of 'non-arbitrary' pertains to salience. For present purposes, 'Property $p$ is salient' means 'Property $p$ is conspicuously different from any other property.' Another sense of 'non-arbitrary' pertains to moral relevance. For present purposes, 'Property $p$ is morally relevant' means 'Property $p$ is moral-standingconferring.' Given this, the preceding claim that there would be no non-arbitrary way of designating which entities have full moral standing and which do not could be understood in one of two ways. It could be understood as the claim that there would be no conspicuous degree of accidental-property possession in terms of which we could designate which things have full moral standing. Or, it could be understood as the claim that there would be no moral-standing-conferring degree of accidentalproperty possession in terms of which we could designate which things have full moral standing.

Now, it should be clear that a conspicuous property is not one and the same as a moral-standing-conferring property - the property of being bipedal is conspicuously shared by individuals (1) - (4), for example, but it is not in itself moral-standing-conferring. Given this, even if a conspicuous degree of accidental-property possession is lacking, it does not follow that there is no degree of accidental-property possession that confers full moral standing. To be sure, if a conspicuous degree of accidental-property possession is lacking, then it may be difficult to determine which things have full moral standing and which don't. But, first, there is no a priori reason to think that establishing which entities have full moral standing and which do not would be anything less than difficult - indeed, there is strong a posteriori reason to think that this is actually very difficult. Second, even

\footnotetext{
${ }^{21}$ See, for example, M.A.Warren. 2000. Moral Status: Obligations to Persons and Other Living Things. New York, NY: Oxford University Press: 148ff.

${ }^{22}$ See David Boonin. 2002. A Defense of Abortion. New York, NY: Cambridge University Press: $39 \mathrm{ff}$.
}

lacking a conspicuous degree of accidental-property possession, it won't be difficult in some cases, such as the case of the standard adult human being (a paradigmatic case of something that has full moral standing if there ever was one) and the case of an inanimate object such as the piece of lint (a paradigmatic case of something that does not have full moral standing if there ever was one).

A second reason defenders of SV have for thinking that intrinsic value does not admit of degrees is as follows. Defenders of SV contend that mere differences in degree of accidental-property possession - no matter how great they may be - is no ground for treating two entities radically differently. As Lee and George write:

But the difference between a being that deserves full moral respect and a being that does not (and can therefore legitimately be disposed of as a means of benefiting others) cannot consist only in the fact that, while both have some feature, one has more of it than the other. A mere quantitative difference (having more or less of the same feature, such as the development of a basic natural capacity) cannot by itself be a justificatory basis for treating different entities in radically different ways. ${ }^{23}$

This set of claims is restated word for word in George's and Tollefsen's book. ${ }^{24}$ And these two sets of claims have more in common than just words: both are left unsupported, as Lee and George (in the former case) and George and Tollefsen (in the latter case) provide no justification whatsoever for these claims. In both cases, it is simply declared that a mere quantitative difference cannot by itself be a justificatory basis for treating different entities in radically different ways. And, needless to say, more is needed here than mere declaration.

Moreover, there is reason to doubt these claims. Consider, for example, two individuals, one who has the basic potential for playwriting (say, a fledgling high-school playwright) while the other has the ultimate potential for playwriting (say, the Pulitzer-prize winning playwright Tony Kushner). Given SV defenders' reasoning, with regard to the potential for playwriting, the difference between these two individuals is merely quantitative. Yet, this mere quantitative difference is grounds for treating them radically differently: it is grounds for awarding the Pulitzer prize to Kushner, producing his plays on Broadway and television, awarding him honorary doctorates (and so on) while, at the same time, not treating the fledgling playwright even remotely similarly.

Or, to use a case directly related to the issue of moral standing, consider two individuals, one who has the basic potential for rational moral agency (e.g. a standard human infant) and one who has the ultimate potential for

\footnotetext{
23 Lee and George 2007, op. cit. note 3, 137.

${ }^{24}$ George and Tollefsen, op. cit. note 3, p. 120.
} 
rational moral agency (e.g. a standard adult human being). Given SV defenders' reasoning, with regard to the potential for rational moral agency, the difference between these two individuals is merely quantitative. Yet, this mere quantitative difference is grounds for treating them radically different: it grounds expecting the standard adult human being to behave responsibly and holding her accountable (through rewards and punishments) for her behavior; being disappointed in her when she behaves immorally; being proud of her when she behaves morally (and so on) while, at the same time, not treating the infant even remotely similarly.

Of course, defenders of SV might claim that, contrary to what I have supposed, these cases do not involve treating the individuals in question radically differently. But this strikes me as implausible. And, in any case, for this to be persuasive, they would need to provide and defend an analysis of 'radically differently'. Thus far, they have not done this and, until they do, we are left to decide for ourselves whether the cases above involve treating the individuals in question radically differently.

\section{On possession of the basic potential for rational moral agency}

The final objection pertains to SV's claim that the essential property the possession of which is sufficient for the wrongness of killing individuals (1) - (4) and, thus, (5) is that of the basic potential for rational moral agency. Of course, for this to be the case, each of these individuals must possess this property. So do they? Let us grant that individuals (4) and (5) do. What about individuals (1) - (3)? Do the standard adult human being, the temporarily comatose adult human being, and the suicidal adult human being possess the basic potential for rational moral agency? They do not. Each has the proximate if not ultimate potential for rational moral agency - each has the active though perhaps not immediately actualizable potential for rational moral agency if not the active and immediately actualizable potential for rational moral agency. None has the basic potential - the active but not remotely actualizable potential - for rational moral agency. To be sure, each of them once had the basic potential for rational moral agency, but none has it anymore. And, given that none of these individuals possesses the basic potential for rational moral agency, the basic potential for rational moral agency cannot be an essential property of the substance sort human organism, despite defenders of SV's declarations to the contrary. Whatever may account for the wrongness of killing individuals (1) - (4), then it cannot be possession of the essential property of the basic potential for rational moral agency.

Defenders of SV might reply to the preceding objection in a number of different ways. First, they might contend that if a human organism possesses a property at any point in its existence, then it possesses that property at every point of its existence and, thus, that individuals (1) - (3) do in fact possess the basic potential for rational moral agency. ${ }^{25}$ But this is simply false - there are many properties that standard adult human beings possess that they did not possess as infants (e.g. the ability to solve new and complex problems). Likewise, there are many properties infants possess that they will not possess as adults (e.g. a not-yet-fully-developed brain). Thus, it's simply not the case that if a human organism possesses a property at any point in its existence, then it possesses that property at every point of its existence.

Second, they might reply that basic, proximate, and ultimate potentialities are just different stages of a single, unified potential. This may be correct descriptively, but in and of itself it entails nothing morally. What's needed here is an argument that these different stages make no moral difference - that this single, unified potential carries the same moral weight regardless of the stage. And, the closest approximation to an argument for this has been discussed already, namely, their declaration that a mere quantitative difference cannot by itself be a justificatory basis for treating different entities in radically different ways. But mere declaration does not an argument make.

And third, they might reply that individuals (1) - (3) formerly possessed the property of the basic potential for rational moral agency and that both the current possession of this property and the former possession of this property suffice for the wrongness of killing individuals (1) - (4). But such a reply would present problems of its own.

First, it would lead to the conclusion that it just as wrong to kill irreversibly comatose adult human beings as it is to kill individuals (1) - (4), a conclusion many will find strongly counterintuitive if not absurd. (Lee and George bite the bullet and accept this conclusion. ${ }^{26}$ I leave it to the reader to decide whether this is a bullet one should bite.)

Second, if the former possession of the basic potential for rational moral agency suffices to give individuals (1) - (4) full moral standing, then human corpses have full moral standing as well. After all, human corpses also formerly possessed the basic potential for rational moral agency. But most people would find the view that human corpses have full moral standing to be absurd.

Finally, the former possession of the basic potential for rational moral agency is a temporally-indexed property: something has the basic potential for rational moral

\footnotetext{
${ }^{25}$ This point is made by David Boonin (Boonin: 50ff) in his rebuttal to what he refers to as the essential property argument, an argument he attributes to Robert E. Joyce, Thomas W. Hilgers, and Paul Ramsey, among others.

${ }^{26}$ Lee and George: 153-154.
} 
agency at a time $\mathrm{t} 2$ if it possessed it at a time $\mathrm{t} 1$. And that temporally-indexed properties can be essential properties is a matter of great controversy, as is evidenced by the relevant literature. ${ }^{27}$

\section{CONCLUSION}

In my initial critique of the substance view, I raised reductio-style objections to the substance view's conclusion that the standard human fetus has the same intrinsic value and moral standing as the standard adult human being, among others. In this follow-up critique, I raised objections to three claims involved in the substance

\footnotetext{
27 See: Sally Haslanger. Endurance and Temporary Intrinsics. Analysis 1989; 49: 119-125; Ryan Wasserman. The Problem of Change. Philosophical Compass 2006; 1(1): 48-57; David Lewis. Tensing the Copula. Mind 2002; 111: 1-13; David Lewis. Rearrangement of Particles: Reply to Lowe. Analysis 2002; 48: 65-72; Josh Parsons. Must a Four-Dimensionalist Believe in Temporal Parts? The Monist 2000; 83: 399-418.
}

view's defense: the claim that the standard human fetus's intrinsic value and moral standing is a function of its potentiality; the claim that the standard human fetus's intrinsic value and moral standing is a function of its essential properties; and the claim that it is the possession of the basic potential for rational moral agency that best accounts for the wrongness of killing the standard human fetus, among others. Both at the level of the premises invoked in support of its conclusion and at the level of the conclusion itself, the substance view is subject to serious objections. As it stands, then, the substance view is simply implausible, all things considered.

Rob Lovering is Assistant Professor of Philosophy at the College of Staten Island/City University of New York. Dr. Lovering's recent publications include 'Does God Know What It's Like Not to Know?' (Religious Studies), 'The Problem of the Theistic Evidentialist Philosophers' (Philo), 'The Ever Conscious View: A Critique' (Philosophy in the Contemporary World), and 'Futures of Value and the Destruction of Human Embryos' (Canadian Journal of Philosophy).' His first book, God and Evidence: Problems for Theistic Philosophers, will be released in Spring 2013 (Bloomsbury) 